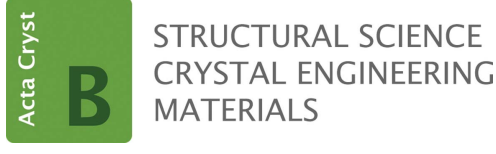

ISSN 2052-5206

Keywords: book review; crystal chemistry.

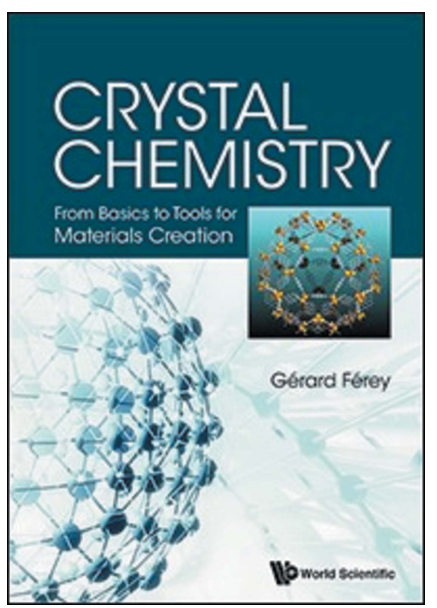

(C) 2017 International Union of Crystallography

\section{Crystal Chemistry. From Basics to Tools for Materials Creation. By Gérard Ferey. World Scientific, 2017, 264 pp. Softcover ISBN 978-981-3144-19-4, price GBP 46.00, hardback, ISBN 978-981-3144-18-7, price GBP 81.00}

\author{
Ulrich Müller* \\ Fachbereich Chemie, Universität Marburg, Hans-Meerwein-Str., 35032 Marburg, Germany. *Correspondence e-mail: \\ mueller@chemie.uni-marburg.de
}

According to the Preface, 'the book aims to be useful for undergraduates', and its title and the list of contents give the impression that this is a textbook for advanced students of chemistry. But that is not the case. Essential concepts of crystal chemistry are not covered at all, such as ionic radius ratios, Pauling's rules, lattice energy, phase diagrams and chemical bonding in solids. Some classes of compounds such as silicates, intermetallics and Zintl phases are completely missing. The conception is not a general building up of knowledge in crystal chemistry, but a notional view at coordination polyhedra and their joining with increasing complexity, reflecting predilections of the author and culminating in results of the author's research.

It is inconsistent that some basic crystallographic terms are explained, such as rotations, rotoinversions, screw rotations and glide reflections, while other crystallographic topics are expected to be known. The terms 'symmetry operation' and 'symmetry element' are used frequently, but not explained, and sometimes confused. Crystallographic group theory, cell settings and cell transformations are not a subject. Point groups and their symbols are not really explained and it is (wrongly) claimed that their number is restricted to 32. Space groups, Hermann-Mauguin symbols and Miller indices are explained in a rather terse way. Rotation axes are termed $A_{2}, A_{3}, \ldots$ and rotoinversion axes $A_{\overline{3}}, A_{\overline{4}}, \ldots$ in lieu of the usual symbols. The use of mathematics is restricted to the calculation of interatomic distances.

Chapter 1 begins with Platonic and Archimedean polyhedra and how they are joined in simple crystal structures (detailed geometric data follow in an appendix). The primitive cubic packing and the close-packings of spheres are explained together with their interstices. An approach that is repeatedly used throughout the book is to insert atoms at the points of contact of the spheres; this is called a decoration or a substitution of joined polyhedra for spheres, 'keeping the topology'. 'Topology' is the all-dominant term in the book, and yet never explained, now and then ambiguous and not quite in accordance with its mathematical definition.

The elaborate Chapter 3 (45 pages) gives instructions on how to interpret perspective drawings, projections and crystal data (lattice parameters, atomic coordinates including symmetry-equivalent positions). This illuminating chapter begins with a lengthy explanation of the rutile structure, which is described as a distorted hexagonal arrangement of 'oxygens' with octahedral interstices occupied by Ti atoms. It is not mentioned that the packing of the oxygen atoms is a tetragonal close packing with the coordination number 11. The structure types of $\mathrm{CaF}_{2}, \mathrm{NaCl}, \mathrm{NiAs}$, cubic and hexagonal $\mathrm{ZnS}, \alpha-\mathrm{Al}_{2} \mathrm{O}_{3}$, $\beta-\mathrm{Ga}_{2} \mathrm{O}_{3}, \mathrm{CdCl}_{2}, \mathrm{CdI}_{2}, \mathrm{MgAl}_{2} \mathrm{O}_{4}$ (spinel), $\mathrm{K}_{2} \mathrm{NiF}_{4}, \mathrm{ReO}_{3}, \mathrm{MoO}_{3}$, tungsten bronzes and several others are explained in detail. That includes their space groups, lattice parameters, atomic coordinates, coordination polyhedra, many figures and the description as packings of spheres with occupied interstices. Ample attention is given to the kinds of connectivity of the coordination polyhedra. The superconductors $\mathrm{YBa}_{2} \mathrm{Cu}_{3} \mathrm{O}_{8-\delta}$ serve as examples to explain the concept of vacancies. 
In Chapter 4, the huge family of the perovskite derivatives is used to discuss the distortions of a highly symmetrical structure. Attention is given to the mutual tilting of the coordination octahedra and to the ordered substitution of cations. This is a clear geometric discussion, but it pays little attention to other aspects (such as symmetry or physical properties). Removing sheets of $\mathrm{O}$ atoms from the $\mathrm{ReO}_{3}$ structure and joining the fragments via edge-sharing octahedra serves to derive the structures of $\mathrm{V}, \mathrm{Nb}, \mathrm{Mo}$ and $\mathrm{W}$ oxides; this is a vivid mental 'mechanism' called a crystallographic shear. Other structures are derived by (mentally) removing part of the $\mathrm{O}$ atoms, resulting in 'defective' perovskites such as $\mathrm{Ca}_{2} \mathrm{Mn}_{2} \mathrm{O}_{5} \square$.

Chapter 5 points out remarkable geometrical similarities between chemically different structures, for example, $\mathrm{Mn}_{2} \mathrm{Hg}_{5}$ and $\mathrm{K}_{0.4} \mathrm{WO}_{3}=\mathrm{K}_{2}\left(\mathrm{WO}_{3}\right)_{5}$, by decorating the $\mathrm{Hg}$ positions with vertex-sharing $\mathrm{WO}_{6 / 2}$ octahedra. The opposite approach is to merge molecular entities to one 'brick' or 'secondary building unit', say three $\mathrm{WO}_{6 / 2}$ octahedra to a 'trimeric' $\left(\mathrm{WO}_{6 / 2}\right)_{3}$ brick, and regarding the structure of a hexagonal tungsten bronze as being built from these bricks. This view is continued in Chapter 6, which resumes the discussion of the perovskites and continues with many more structures.

In Chapter 7 the size of the secondary building units is increased, for example, by joining tetrahedra to super-tetrahedra, say to $\left[\mathrm{In}_{10} \mathrm{Se}_{16} \mathrm{Se}_{4 / 2}\right]^{6-}$, and then connecting their vertices to a framework, leaving pores in between. The pores can host appropriate counter-ions and the secondary building units can be connected by spacers. This assembly, called 'the scaling up concept', can actually be performed experimentally by crystallizing an inorganic material from solution in the presence of well chosen counter-ions or organic linkers acting as spacers. The contents of the pores can be removed or exchanged. The art of preparing such materials with tailored pores is described. The last sections of the book give thoughts to possible applications of such porous materials.
The large number of figures is worthy of praise and, in general, the quality of the artwork is excellent. Unfortunately, a number of figures have been scaled down to an extent that it becomes hard to recognize anything.

Repeatedly, there are not quite correct assertions. For example, 'only axes of orders 2, 3, 4, 5 and 6 are encountered in the three-dimensional space'; 'inverse axial symmetry is obtained by adding a center of symmetry to an axis'; 'two perpendicular twofold axes generate a symmetry center'; stating that the threefold axis of a rhombohedral structure is not a crystallographic axis. The $\mathrm{O}$ atoms of rutile are said to be in a general position, although they are in the special position $4 f$; to assert that $\mathrm{Cu}_{2} \mathrm{O}$ is the antitype of cristobalite- $\mathrm{SiO}_{2}$ is an inexcusable error.

There are only very few misprints, such as a wrong Wyckoff letter in Table 12.1 and the missing chemical formulae in Fig. 3.36. Linguistic lapses are frequent, for example, 'the nickel octahedra' (meaning coordination octahedra around $\mathrm{Ni}$ ), 'two-dimensional solid' (a solid is always three-dimensional), 'the cell becomes more tetragonal' and 'tetrahedral layer'. The term 'mechanism' is constantly used when talking of a mentally imagined rearrangement. The word 'identical' is used with differing meanings: 'equal', 'of the same kind', 'with a similar arrangement' or 'congruent when projected perpendicularly onto each other'. To some degree, the text is Frenglish, and there are words taken over from French, such as 'helicoidal axis' (screw axis) or 'particular position' (special position).

To summarize: this is not a book for those who want to learn the principles of crystal chemistry. In addition, huge amounts of particular structures overburden the student. The text gives an impression of the diversity of solid-state chemistry, mainly regarded as geometrical compositions of polyhedra. In essence, the book is a review article of the author's impressive contributions to research, preceded by a six-chapter introduction. 